\title{
Endoscopic Vascular Decompression for the Treatment of Trigeminal Neuralgia: Clinical Outcomes and Technical Note
}

This article was published in the following Dove Press journal: Journal of Pain Research

\section{Zhixiang Sun \\ Yu Wang \\ Xintao Cai \\ Shan Xie (D) \\ Zhiquan Jiang}

Department of Neurosurgery, The First Affiliated Hospital of Bengbu Medical College, Bengbu 233000, People's Republic of China
Correspondence: Zhiquan Jiang Department of Neurosurgery,

First Affiliated Hospital of Bengbu Medical College, 287 Changhuai Road, Bengbu, Anhui 233004, People's Republic of China Tel +86-I396607597I

Email bbjiangzhq@।63.com
Purpose: Microvascular decompression (MVD) surgery is considered as an effective method with which to treat trigeminal neuralgia (TN). However, sometimes MVD surgery fails due to incomplete decompression of the responsible vessels caused by a poor visual field. In this study, we evaluated the benefits of endoscopic visualization and the value of full endoscopic vascular decompression (EVD) by describing the surgical results of 20 patients with TN after EVD.

Patients and Methods: This was a retrospective study in a single institution of 20 patients with TN who received EVD between April 2018 and October 2019. All patients underwent EVD via the suboccipital retrosigmoid approach without microscopy at any stage. Abnormal muscle response (AMR) and brainstem auditory evoked potentials (BAEPs) were routinely monitored throughout the procedure. Follow-up was conducted by outpatient and telephone interviews. The degree of facial pain was graded using the Barrow Neurological Institute (BNI) pain intensity score; a BNI of 1 was considered as the best result while a BNI of 2 or 3 was considered as a satisfactory result. Follow-up time ranged from 8 to 24 months, with a mean of $18 \pm 4.36$ months.

Results: All 20 patients with severe preoperative pain (BNI of 5) achieved immediate relief or complete control of pain after surgery (BNI of 1 to 2). Vascular conflicts were observed during surgery in all of the patients. None of the patients experienced hearing loss, facial paralysis, intracranial infection, cerebrospinal fluid leakage, cerebral hemorrhage, or death, following the operation.

Conclusion: When carried out by surgeons with endoscopic experience, EVD can provide a clear surgical field of view and reduce the risk of surgical injury. Our findings indicate that EVD is a safe and effective surgical method for the treatment of TN.

Keywords: trigeminal neuralgia, endoscopic, microvascular decompression

\section{Introduction}

Trigeminal neuralgia (TN) is a transient and paroxysmal severe pain in the trigeminal nerve distribution area on one side of the face. The predominant pathophysiological mechanism of $\mathrm{TN}$ is demyelination of the trigeminal nerve root in the cerebellopontine angle (CPA) area caused by neurovascular compression (NVC). ${ }^{1,2}$ At present, microvascular decompression (MVD) is recognized as the most effective surgical method with which to treat primary $\mathrm{TN}^{3,4}$ The key to maximizing the success rate of this form of compression is the accurate location of the compression region, particularly the root entry zone (REZ). ${ }^{5}$ The development 
of microsurgery has led to gradual improvements in the success rate of MVD. However, not all vascular conflicts can be detected microscopically, particularly when the responsible vessels are in the anterior part of the nerve. Endoscopes provide panoramic views and bright lighting and can therefore identify and manage vascular conflicts much more effectively than traditional methods. ${ }^{6}$ An increasing number of institutions have now adopted endoscopes as the standard auxiliary tool for conventional MVD. However, very few reports involving MVD have described the complete application of endoscopes. ${ }^{7-9}$ In this study, we investigated the surgical results of endoscopic vascular decompression (EVD) in our institution and discussed our surgical experience of EVD.

\section{Patients and Methods}

\section{Clinical Materials}

We performed a retrospective study of 20 patients with TN who received EVD from the same surgeon in our hospital between April 2018 and October 2019. The baseline characteristics of the 20 patients are shown in Table 1 . All patients with typical facial pain were evaluated by contrast-enhanced magnetic resonance imaging (MRI) to exclude sinus inflammation, intracranial tumor, multiple sclerosis, and other major factors. MRI or magnetic resonance tomographic angiography (MRTA) was used to detect the responsible vessel (Figure 1A and B). All patients signed a consent form prior to the operation and

Table I Baseline Characteristics of Patients

\begin{tabular}{|l|l|l|}
\hline Age & Min-max & $48-7 I$ \\
& Average & 55 \\
Pender & Female & $12(60 \%)$ \\
& Male & $8(40 \%)$ \\
& VI & 0 \\
& V2 & $I I(55 \%)$ \\
& V3 & $3(15 \%)$ \\
& VI, V2 & $I(5 \%)$ \\
& V2, V3 & $4(20 \%)$ \\
Site of operation & VI, V2, V3 & $I(5 \%)$ \\
& Right & $I I(55 \%)$ \\
& Left & $9(45 \%)$ \\
& SCA & $I 4(70 \%)$ \\
& AICA & $2(10 \%)$ \\
& Vein only & $I(5 \%)$ \\
& SCA and AICA & $2(10 \%)$ \\
& SCA and vein & $I(5 \%)$ \\
\hline
\end{tabular}

Abbreviations: SCA, superior cerebellar artery; AICA, anterior inferior cerebellar artery.

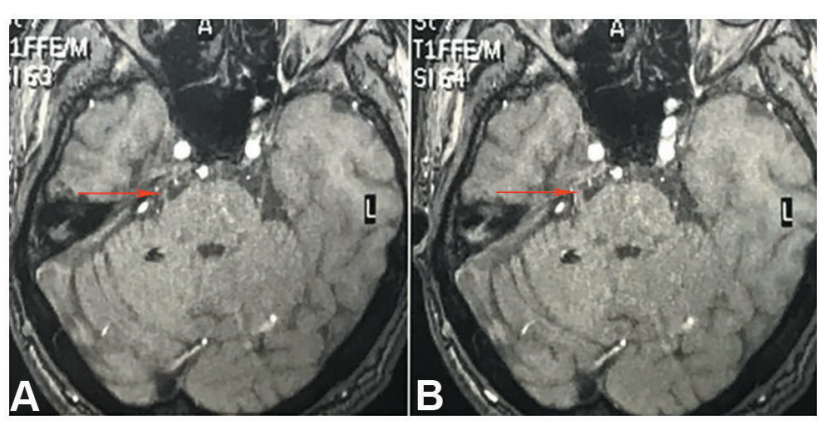

Figure I Axial magnetic resonance imaging (MRI) showing a blood vessel compressing the root entry zone (REZ) of the trigeminal nerve at the brainstem ( $\mathbf{A}$ and $\mathbf{B}$; red arrow).

received full EVD; microscopy was not used at any stage of the operation. Follow-up time ranged from 8 to 24

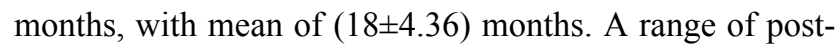
operative conditions were recorded, including pain relief, cerebrospinal fluid (CSF) leakage, intracranial infection, cerebral hemorrhage, and neurological deficits. The degree of relief for facial pain was graded by the Barrow neuropathic pain intensity score (BNI). All patients were followed up by regular telephone or outpatient visits after discharge, to assess whether the pain had been relieved or had disappeared, whether the pain had recurred and whether there were other neurological complications.

\section{Surgical Techniques}

First, the hair on the head was completely removed, and general anesthesia was applied. We then used a Mayfield head frame to fix the head onto the operating bed. The patient was placed in a lateral decubitus position with the healthy side facing downwards. The head was lowered by $15^{\circ}$, and rotated $10^{\circ}$ to the healthy side, so that the mastoid was at the highest position and the blood flow in the neck was not obstructed. During the procedure, we routinely monitored abnormal muscle response (AMR) and brainstem auditory evoked potentials (BAEPs). A skin incision was made behind the ear of (approximately $3 \mathrm{~cm}$ in length) to create a $2 \mathrm{~cm}$ opening in the bone (Figure 2A and B). Next, we opened the dura mater at a position posterior to the junction of the sigmoid and the transverse sinus. Once the dura mater had been cut, all subsequent procedures were carried out with an endoscope but without microscopy.

First, we opened the subarachnoid space and slowly released the cerebrospinal fluid; we used small pieces of cerebral cotton to provide sufficient operating space for the endoscope. At this time, we used a rigid $30^{\circ}$ endoscope 


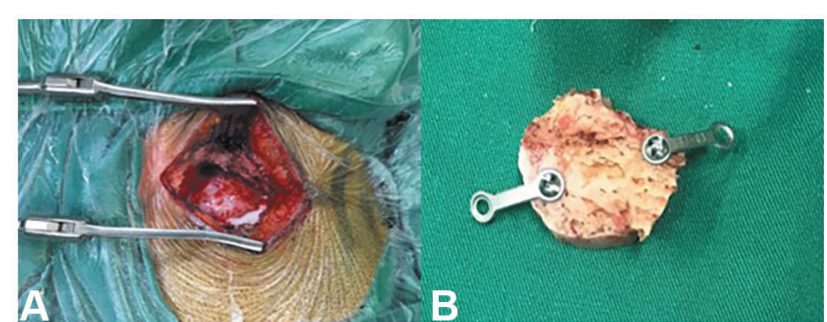

Figure $2 \mathrm{~A}$ skin incision was made behind the ear (approximately $3 \mathrm{~cm}$ in length) and a $2 \mathrm{~cm}$ bony opening was created (A). A bone flap approximately $2 \mathrm{~cm}$ in diameter (B).

with an outside diameter of $4 \mathrm{~mm}$ (Karl Storz, Tuttlingen, Germany); when combined with a high-definition camera, this endoscope provides excellent visualization. By adjusting the angle of the $30^{\circ}$ endoscope, it was possible to observe any hidden structure within the operating area without having to retract the brain. The endoscope was attached to the operating table using a Mingjie Minimally Invasive Surgical Robot (Deming, DR0502, Hefei, Anhui, China) (Figure 3A and B); this released our hands so that we could concentrate on fine dissection. We believe that one of the major technical challenges to EVD is the potential risk of instruments clashing with the endoscope within the limited scope of cerebellopontine angle (CPA). By maintaining a strict triangle during the introduction and placement of the endoscope and the right- and left-hand instruments, we were able to minimize confusion and conflict between instruments within the tight confines of the CPA. The endoscope was placed at the vertex of a virtual equilateral triangle that was constructed in the surgical field of view; the vertex represented the " 12 o'clock" position. The aspirator and micro-instrument passed underneath the endoscope at the "5 o'clock" and

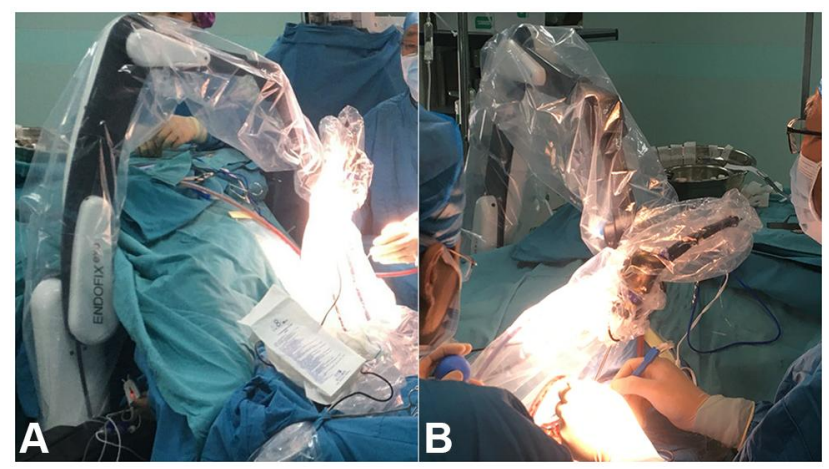

Figure 3 A Mingiie minimally invasive surgical robot. This system features a onebutton operating system with an electromagnetic sensing control fixes endoscope for surgery (A and $\mathbf{B})$.
"7 o'clock" positions. In this way, we were able to maximize surgical safety while maintaining good operability.

Once we had entered the CPA, we identified the trigeminal nerve. Next, we released the nerve by fully dissecting the surrounding arachnoid membrane. At the point at which the petrosal vein blocked the visual field on the surgical path (Figure 4A), we separated the surrounding arachnoid membrane, thus allowing us to completely dissect and free the vein. By adjusting the angle of the endoscope, the lens was able to cross the vein and observe specific points of interest. At this stage in the operation, it was important for us to protect the facial nerve and pull the vein only when necessary. Because the operating space was very narrow, it was sometimes necessary to cut off the petrosal vein. However, once the petrosal vein was sacrificed, it was important to monitor the cerebellum for swelling post-operatively. When dissecting and separating arachnoid membranes, it is possible to directly coagulate any hemorrhage arising from the small veins. Bleeding from the petrosal vein is more difficult to control, because in such situation, the endoscope lens rapidly becomes stained with blood. During such situations, we frequently wash the endoscope lens and operative area with saline. An aspirator is then used to suck out the contaminated saline so that the field-of-view can be cleared. Fluid gelatin (Johnson, New Jersey, American) can also be injected into the bleeding point. A gelatin sponge should also be attached to the outside of the bleeding point instead of trying to coagulate the vein. Potential vascular conflicts were identified by $360^{\circ}$ observation of the $\mathrm{TN}$ and its surroundings, especially the subarachnoid, RZE, and Meckel's cavities.

Once the vascular conflict had been identified, we fully dissociated the responsible vessel and used a Teflon felt to decompress the conflicting vessel. We re-surveyed the surgical field carefully to ensure that we had fully dealt with all responsible vessels before ending the operation.

It is interesting to note that we encountered one case involving an intraneural vessel. Thus had to be sacrificed because the vein prevented us from exposing the trigeminal nerve (Figure 4B). In this case, the responsible blood vessel was the superior cerebellar artery that passes through the trigeminal nerve (Figure 4C). In this case, we dissected the arachnoid adherence around the artery so that the artery could be fully mobilized. Next, we placed a Teflon pledget into the gap between the artery and the nerve root (Figure 4D). We frequently irrigated the entire operative field as this eliminates debris, ensures that the visual field remains clear, 


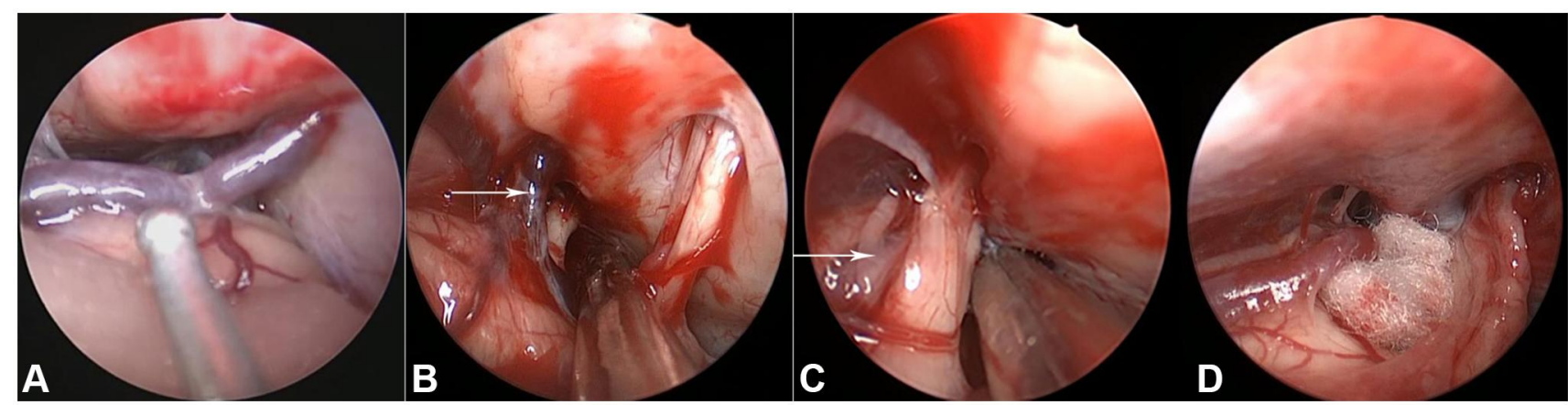

Figure 4 An image showing the petrosal vein blocking the visual field of the surgical path. (A) Right-sided approach demonstrating a vein (white arrow) that prevented us from exposing the trigeminal nerve (B). The superior cerebellar artery (white arrow) passing through the trigeminal nerve (C). Separation of the superior cerebellar artery from the nerve and a Teflon pledget was interposed into the gap between the artery and the nerve root (D).

and reduces the risk of thermal damage to the brain. We also repeatedly irrigated the surgical cavity to flush out bloodstained CSF and to check whether the Teflon pledget had been displaced. Finally, the endoscope was removed and the dura mater was sutured so that it was impermeable to water. Next, the bone flap was fixed with a titanium chain, bone cement was used to fill the skull defect, and absorbable sutures were used to close the skin.

\section{Results}

A total of 20 patients with TN underwent complete endoscopic vascular decompression during the period of research. The vessels responsible for compression are reported in Table 1. A total of 23 vessels were identified in the 20 patients, including 17 superior cerebellar arteries (SCAs; 73.91\%; Figure 5A), 4 anterior inferior cerebellar arteries (AICAs; $17.39 \%$; Figure 5B), and 2 venous vessels $(8.70 \%$; Figure 5C). Single vessel conflict was found in 17 cases $(85 \%)$, including 14 cases $(82.35 \%)$ associated with the SCA, 2 cases (11.76\%) associated with the AICA, and 1 case $(5.88 \%)$ associated with a venous blood vessel. Three cases (15\%) involved double vessel conflict, 2 cases (75\%) involved the SCA and the AICA, and 1 case (25\%) involved the SCA and a vein. The site of conflict was located at the nerve root entry zone in 18 patients $(90.00 \%)$ and at Meckel's cave in 2 patients $(10.00 \%)$. We also observed two vessels (8.69\%) lying anterior to the nerve and one intraneural vessel (4.35\%); it would have been relatively easy to miss these vessels with a microscope. It is far more complex to observe and dissect such blood vessels under a microscope; this form of retraction is likely to cause swelling in the cerebellum swelling and induce nerve function damage.

The preoperative BNI score for all patients was 5 . Following surgery, 16 patients $(80 \%)$ achieved significant remission and had a BNI of 1; the remaining 4 patients (20\%) achieved good remission and had a BNI of 2. One patient's heart rate slowed down during surgery when we were separating the vascular conflict. The operation was

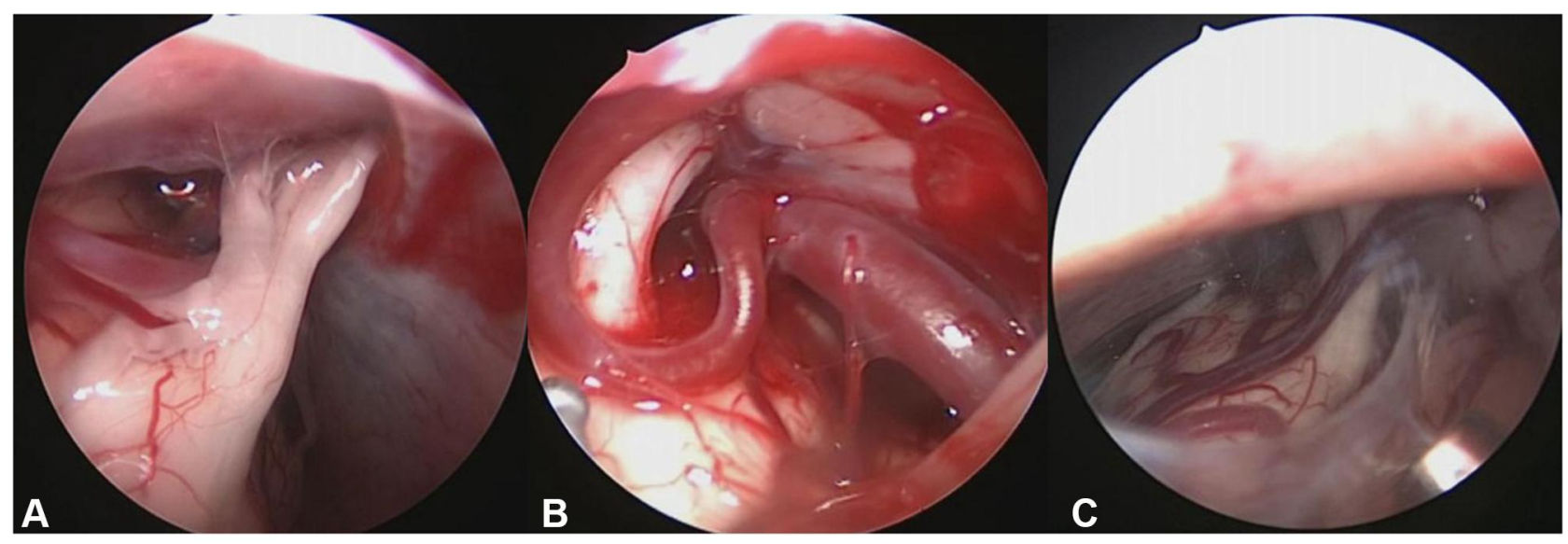

Figure 5 An endoscopic view revealing compression of the trigeminal nerve by the SCA (A). An endoscopic view showing compression of the trigeminal nerve by the AICA (B). An endoscopic view showing compression of the trigeminal nerve by a vein (C). 
paused, while we administered atropine and waited for the heart rate to recover.

There were no adverse reactions after surgery. One patient developed a herpetic spat on the third day after surgery but this subsided after one week of treatment with neurotrophic drugs. One other patient developed dizziness and one patient developed mild facial numbness; these conditions both disappeared gradually during the followup period. There were no postoperative intracranial infections, cerebrospinal fluid leakage, cerebral hemorrhage, or death. The follow-up period was 8-24 months, with a mean of $(18 \pm 4.36)$ months. None of the patients experienced any pain.

TN is common facial pain syndrome. MVD is the first choice treatment for patients with primary TN who find it difficult to tolerate pain. ${ }^{3-5,10-13}$ Usually, we perform MRI and MRTA prior to surgery to observe the relationship between the trigeminal nerve and the peripheral blood vessels. Using these methods, we successfully identified a responsible vessel prior to surgery for all 20 of our patients. However, once visualized with an endoscope, we found that three patients had a double vessel conflict; thus, our pre-surgical accuracy rate for detection was $85 \%$. In a recent study of 449 trigeminal nerve patients, Hao et al reported that the sensitivity of MRTA was $95.8 \%$ (430/449) and the specificity was $71.0 \%(22 / 31) .{ }^{14}$ These data show that direct intraoperative visualization is still the most effective procedure for determining vascular conflicts. Traditional forms of MVD that are carried out under a microscope have achieved good clinical results. However, the field of view for microscopes under such circumstances is limited to the dural opening. This is because microscopes require a straight line from the lens to the point of interest to achieve the best visualization. This means that to fully identify vascular conflicts by microscopy, we need to create larger bone openings, or retract the cerebellum or brainstem to greater extents. In contrast, an endoscope can provide an excellent view inside the CPA through a small opening in the dura because it requires divergent distal light. Therefore, compared with microscopes, endoscopes are much more efficient at identifying hidden vascular conflicts, such as in the REZ or Meckel's cave.

In a previous study, Chen et al studied 167 patients with trigeminal neuralgia and diagnosed $85.26 \%$ of neurovascular conflicts using only a microscope. ${ }^{15}$ The remaining $14.76 \%$ of cases were only diagnosed with the assistance of an endoscope. In our case, there are two vascular conflicts in front of the nerve; these could easily have been missed by the microscope but readily observed using an angled endoscope. In another study, Tang et al found that a $30^{\circ}$ endoscope provided a field-of-view that was almost two-fold larger than that provided by a microscope. ${ }^{16}$ Similarly, we also recommend the use of a $30^{\circ}$ endoscope rather than a $0^{\circ}$ endoscope; using the $30^{\circ}$ endoscope, it is only necessary to adjust the angle slightly to observe hidden anatomy, such as nerve fronts and Meckel's cave. The use of an endoscope allows panoramic views and good visualization of vascular conflicts without the need to retract brain tissue and nerve. Similarly, the use of an angled endoscope can prevent us from sacrificing the petrosal vein and thus prevent swelling in the cerebellum swelling caused by postoperative venous reflux disorder. We did not encounter any serious or permanent complications in our series of patients when using the EVD technique. The existing literature also report a low incidence of complications when using the EVD technique and no endoscope-related complications.

Bohman et al reported that $94 \%$ of 47 patients undergoing EVD for TN experienced some form of pain relief. ${ }^{17}$ Furthermore, these authors reported a relatively low rate of complications. None of the complications were permanent. One patient had hearing loss and one patient experienced postoperative CSF leakage. In 2014, Setty et al analyzed the results of a series of patients that were treated completely by EVD. ${ }^{18}$ Of the 57 patients investigated, 56 (98\%) reported complete relief or well-controlled pain after surgery; the complication rate was $4 \%$. In another study, Dubey et al reported no serious complications; $93.5 \%$ and $82.6 \%$ of patients had complete pain relief at 3 months and 5 years follow-up, respectively. ${ }^{19}$

In the present study, we found that all 20 cases (100\%) experienced pain relief. Furthermore, none of the patients experienced a recurrence of pain during a mean of (18 \pm 4.36 ) months of follow-up. It is possible that this may have been due tour method of case selection as we only included cases involving typical TN. In a previous study, Xiang et al reported a prospective study of 213 patients with TN who randomly received EVD or MVD. ${ }^{20}$ Furthermore, these authors stated that there was no significant difference in the cure rates, or the incidence of surgical complications, when compared between neuroendoscopy and microscopy. In another study, Lee et al reported that both the full EVD technique and conventional MVD appear to provide patients with the same levels of relief with regards to facial pain. ${ }^{8}$ When 
compared between the two groups, the incidence of complications was similar, except that the incidence of headache in the EVD group was significantly lower at one month postoperatively.

The fully endoscopic technique does have some disadvantages that need to be considered, including poor depth of field and the need for hand-eye coordination. This requires a significant amount of practice on models and cadavers. However, once the steep learning curve has been overcome, the entire procedure becomes easy and safe. Furthermore, we must consider that decompression is dependent on bimanual dexterity; this level of dexterity cannot be achieved if the surgeon is forced to hold the endoscope. Therefore, we prefer to use an endoscopeholder for full EVD so that the surgeon can complete the decompression freely without the need for a surgical microscope. However, the endoscope-holder does not provide a satisfactory dynamic range of movement. According to our experience, it is better to carry out EVD procedures with an assistant who has significant endoscopic experience; by synchronizing with the main operator, the presence of an assistant, can significantly shorten the operative time.

There are some limitations associated with our study that need to be considered. For example, our study only reported a small number of patients; the microscopic technique has been reported in a large number of patients. In addition, our follow-up period was very short. At present, we are conducting a prospective cohort study of EVD and MVD so that we can further evaluate the safety and efficacy of EVD.

\section{Conclusion}

Endoscopic microvascular decompression is a safe and effective procedure that can be used to cure TN, provided that the surgeon involved is experienced in endoscopic surgery. Compared with traditional microscopy, the endoscope has a number of advantages that can help to improve clinical outcomes. Our findings require further confirmation in a larger cohort of patients.

\section{Ethics Approval and Consent to Participate}

The study was approved by the Research Ethics Committee of Bengbu Medical College. The need for informed consent was waived by the Research Ethics Committee because the study was retrospective. Under the Declaration of Helsinki, patient data was maintained with confidentiality.

\section{Funding}

This study is supported by the Natural Science Foundation of Anhui Province (KJ2018A0995).

\section{Disclosure}

The authors report no conflicts of interest in this work.

\section{References}

1. Love S, Coakham HB. Trigeminal neuralgia: pathology and pathogenesis. Brain. 2001;124(12):2347-2360.

2. Moller AR. Vascular compression of cranial nerves: II: pathophysiology. Neurol Res. 1999;21(5):439-443.

3. Barker FG, Jannetta PJ, Bissonette DJ, et al. The long-term outcome of microvascular decompression for trigeminal neuralgia. New Engl J Med. 1996;334(17):1077-1083.

4. Lovely TJ, Jannetta PJ. Microvascular decompression for trigeminal neuralgia. Surgical technique and long-term results. Neurosurg Clin N Am. 1997;8(1):11-29.

5. Haines SJ, Jannetta PJ, Zorub DS. Microvascular relations of the trigeminal nerve. An anatomical study with clinical correlation. J Neurosurg. 1980;52(3):381-386.

6. Broggi M, Ferroli P, Acerbi F, et al. The value of endoscopy in microvascular decompression procedures. Neurosurg. 2012;71(2): E564.

7. Jarrahy R, Eby JB, Cha ST, et al. Fully endoscopic vascular decompression of the trigeminal nerve. Minim Invasive Neurosurg. 2002;45 (01):32-35.

8. Lee JYK, Pierce JT, Sandhu SK, et al. Endoscopic versus microscopic microvascular decompression for trigeminal neuralgia: equivalent pain outcomes with possibly decreased postoperative headache after endoscopic surgery. $J$ Neurosurg. 2017;126(5):1676-1684.

9. Kabil MS, Eby JB, Shahinian HK. Endoscopic vascular decompression versus microvascular decompression of the trigeminal nerve. Minim Invasive Neurosurg. 2005;48(04):207-212.

10. Sharma R, Phalak M, Katiyar V, et al. Microvascular decompression versus stereotactic radiosurgery as a primary treatment modality for trigeminal neuralgia: a systematic review and meta-analysis of prospective comparative trials. Neurol India. 2018;66(3):688-694.

11. Wang X, Wang H, Chen S, et al. The long-term clinical outcomes of microvascular decompression for treatment of trigeminal neuralgia compressed by the vertebra-basilar artery: a case series review. $B M C$ Neurol. 2019;19(1):217.

12. Zhao H, Wang X, Zhang Y, et al. Management of primary bilateral trigeminal neuralgia with microvascular decompression: 13-case series. World Neurosurg. 2018;109:e724-e730.

13. Koopman JS, de Vries LM, Dieleman JP, et al. A nationwide study of three invasive treatments for trigeminal neuralgia. Pain. 2011;152 (3):507-513.

14. Hao YB, Zhang WJ, Chen MJ, et al. Sensitivity of magnetic resonance tomographic angiography for detecting the degree of neurovascular compression in trigeminal neuralgia. Neurol Sci. 2020. doi:10.1007/s10072-020-04419-0

15. Chen MJ, Zhang WJ, Yang C, et al. Endoscopic neurovascular perspective in microvascular decompression of trigeminal neuralgia. J Craniomaxillofac Surg. 2008;36(8):456-461.

16. Tang CT, Baidya NB, Ammirati M. Endoscope-assisted neurovascular decompression of the trigeminal nerve: a cadaveric study. Neurosurg Rev. 2013;36(3):403-410. 
17. Bohman LE, Pierce J, Stephen JH, et al. Fully endoscopic microvascular decompression for trigeminal neuralgia: technique review and early outcomes. Neurosurg Focus. 2014;37(4):E18.

18. Setty P, Volkov AA, D'Andrea KP, et al. Endoscopic vascular decompression for the treatment of trigeminal neuralgia: clinical outcomes and technical note. World Neurosurg. 2014;81 (3-4):603-608.
19. Dubey A, Yadav N, Ratre S, et al. Full endoscopic vascular decompression in trigeminal neuralgia: experience of 230 patients. World Neurosurg. 2018;113:e612-e617.

20. Xiang H, Wu G, Ouyang J, et al. Prospective study of neuroendoscopy versus microscopy: 213 cases of microvascular decompression for trigeminal neuralgia performed by one neurosurgeon. World Neurosurg. 2018;111:e335-e339.

\section{Publish your work in this journal}

The Journal of Pain Research is an international, peer reviewed, open access, online journal that welcomes laboratory and clinical findings in the fields of pain research and the prevention and management of pain. Original research, reviews, symposium reports, hypothesis formation and commentaries are all considered for publication. The manuscript

Submit your manuscript here: https://www.dovepress.com/journal-of-pain-research-journa management system is completely online and includes a very quick and fair peer-review system, which is all easy to use. Visit http:// www.dovepress.com/testimonials.php to read real quotes from published authors. 